\title{
Modern trends in applied probability: an additional paper in the collection of overview papers
}

This volume includes an invited overview paper as part of a special issue which arose out of the 20th INFORMS-APS conference. The conference was held in Brisbane, Australia, in July 2019, and the next edition is scheduled to be run in Nancy, France, in 2022. Following the Brisbane conference, a call to prepare overview papers on modern trends in applied probability was made and several overview papers have already appeared in this journal. These include an overview by Kristy Gardner and Rhonda Righter dealing with product form FCFS systems; an overview by Jinsheng Chen, Jing Dong, and Pengyi Shi dealing with skill-based routing; and an overview by Azam Asanjarani, Yoni Nazarathy, and Peter Taylor dealing with parameter and state estimation in queues.

The overview paper included in this volume is by Moshe Haviv and Liron Ravner. It presents a comprehensive survey of queueing models with strategic timing of arrivals. In such models, the arrival time is a decision variable available to the customers and the joint behaviour of all customers determines the performance of the system. In many interesting cases, a game-theoretic formulation of the system allows us to describe the equilibrium arrival time distribution of all customers. Such analysis involves transient analysis of the underlying system coupled with game-theoretic considerations. In recent years, there have been dozens of research papers dealing with variants of these types of strategic arrival models. The current overview paper presents various models and results of the field in a unified manner.

Guest Editors: Ross McVinish, Yoni Nazarathy, and Giang Nguyen.

Publisher's Note Springer Nature remains neutral with regard to jurisdictional claims in published maps and institutional affiliations. 